Pathologe 2008 • [Suppl 2] 29:381-382 DOI 10.1007/s00292-008-1020-4

Online publiziert: 7. September 2008

(c) Springer Medizin Verlag 2008

\section{G. Haroske}

Institut für Pathologie, Krankenhaus Dresden-Friedrichstadt

\title{
Sitzungsbericht der AG Informatik der DGP
}

\author{
Berlin, 15. Mai 2008
}

\section{Ein „,klassisches“" Thema der AG Infor- matik, die Telepathologie, fand als ein Hauptthema „Virtuelle Mikrosko- pie" der Tagung der DGP großen Zu- spruch über die AG Informatik hinaus und wurde in mehreren Vorträgen auch während der AG-Sitzung aus- führlich diskutiert.}

Vor wenigen Jahren noch als Illusion angesehen, ist die virtuelle Mikroskopie heute bereits dabei, sich in der studentischen Ausbildung zu etablieren. Insbesondere in Basel, Heidelberg und Aachen wurden sehr gute Erfahrungen mit dem Einsatz der virtuellen Mikroskopie in den histopathologischen Kursen gemacht, wobei durch die Einbindung in E-learningPlattformen (Glatz K et al., http://pathorama.ch, Andrulius $\mathrm{M}$ et al., Open Source Lernplattform Moodle, Sinn P, Hauptvortrag Sa-042) bzw. die Verknüpfung mit "Video Podcasting" (Perez-Bouza A et al., http://www.pathocast.blip.tv) sowohl der Umgang mit WSI (,Whole Slide Images" = "Virtual Slides") durch die Lehrenden erheblich erleichtert wird, als auch die Akzeptanz dieser Darstellungsart durch die Studierenden, auch entgegen pessimistischer Erwartungen, sehr hoch ist. Insbesondere die enge Verzahnung zwischen klinischen, histologischen und makroskopischen Befunden wird durch die Studenten hoch geschätzt.

Mit den gleichen Werkzeugen werden auch WSI im Rahmen klinisch-pathologischer Konferenzen bereits praktisch effektiv eingesetzt (Andrulis $M$ et al.).

Dagegen kommt der Einsatz der virtuellen Mikroskopie in der Weiterbildung der Pathologen, zumindest in Deutschland, nur zögernd voran. Während in der Schweiz sich Pathorama.ch auch als Plattform für IAP-Weiterbildungen und Kurse der Schweizerischen Gesellschaft für Pathologie bewährt, wird das deutsche Pendant (https://iap.virtuelle-mikroskopie. de) bisher nur zögerlich angenommen, obwohl eine große Zahl der IAP-Lehrserien bereits als WSI zur Verfügung steht. Es wird zunehmend klarer, dass nicht die noch sehr teure Technik zur Herstellung von WSI, sondern die Verbindung der Bilder mit plattform- und browserunabhängigen Verwaltungs- und Präsentationswerkzeugen über Akzeptanz und Brauchbarkeit dieser Technologie im "Alltag“ der Fort- und Weiterbildung sowie in der Konsultationspathologie entscheidet. Bisher stoßen alle proprietären Lösungen der Hersteller von virtuellen Mikroskopiesystemen an eben diese Grenzen.

Die technologischen und logistischen Herausforderungen an einen Routinebetrieb der virtuellen Mikroskopie, beispielsweise in der Konsultations- und Zweitmeinungspathologie, sind noch enorm und werden erst mittelfristig gelöst werden können. In diesen Kontext sind auch die Arbeiten aus Magdeburg (Roessner A et al., Zwönitzer R et al., Kalinski $T$ et al.), Berlin (Hufnagl P, Hauptvortrag Sa-022, Müller B, Hauptvortrag Sa-024) und Freiburg (Kayser G, Hauptvortrag Sa-023) einzuordnen, die sich mit speziellen Aspekten der methodischen Entwicklung der virtuellen Mikroskopie beschäftigen, z. B. der Erschließung der 3. Dimension in WSI oder der Einbindung von Bildanalyselösungen zur Ver- feinerung der Aufnahmetechnik oder der automatischen Auswertung großer Mengen von WSI.

Wegen der diesjährigen Konzentration auf das Thema der virtuellen Mikroskopie traten Vorträge zur "klassischen Telepathologie" quantitativ etwas in den Hintergrund, boten aber aufschlussreiche Erfahrungsberichte aus praktischen Anwendungen im Lande und in einem Entwicklungshilfeprojekt in Tansania. Der Vortrag von Bertolini J et al., Leipzig, beschäftigte sich mit den Resultaten von mehr als 500 Schnellschnitt-Telepathologie-Sitzungen zwischen Zwickau und Leipzig, die sowohl hinsichtlich der Qualität (keine falsch-positive Diagnose, 1 falsch-negative Diagnose im Falle eines Schilddrüsenkarzinoms) als auch der „turn around time" zwischen 7 und 30 min der konventionellen intraoperativen Schnellschnittdiagnostik in nichts nachsteht. Völker $\mathrm{H}-\mathrm{U}$ et al., Würzburg, zeigten die Ergebnisse von 300 Fällen aus Tansania, bei denen die Diagnosen aus statischer Telepathologie und den zugrunde liegenden Originalschnitten verglichen wurden. Wie aus ähnlichen Untersuchungen aus Südostasien und Ozeanien in den Vorjahren berichtet, war die diagnostische Qualität hoch, nicht akzeptable (behandlungsrelevante) Diskrepanzen fanden sich bei weniger als 2,5\% der Fälle. Ursächlich wurden dafür fast ausschließlich Ausbildungsmängel des lokalen Personals identifiziert. Für Entwicklungsländer sind derartige Projekte eine enorme Hilfe, gestatten sie doch sowohl die Behandlung von Erkrankungen, die ohne eine zeitnahe histopathologische Diagnostik nicht behan- 
delt werden würden, als auch die nachhaltige Fort- und Weiterbildung der Pathologen und des Laborpersonals vor Ort. In 2 Vorträgen aus Münster (Korsching $E$ et al.) wurden biostatistische Lösungen für die Analyse von Daten aus „tissue microarrays" (TMA) vorgestellt. Derartige Analysen aus sog. „High-throughputVerfahren“ erlangen zunehmendes Interesse, sind aber für Pathologen oft schwer nachvollziehbar. Vorgestellt wurden daher Lösungen, die Schritt für Schritt entlang statistischer Algorithmen mittels des weit verbreiteten Open Source Statistikpakets R entwickelt werden können. Im Anschluss an die AG-Sitzung wurde deshalb auch noch ein Workshop zur Einführung von $\mathrm{R}$ für Pathologen angeboten (Korsching $E)$.

Schrader $T$ et al. berichteten über Entwicklungsfortschritte im Open European Nephrology Science Center, einem DFGgeförderten Forschungsinformationzentrum, bezüglich der automatischen Qualitätssicherung von digitalen klinischen und pathologischen Daten unter Einbeziehung von WSI. Die Lösung dieser sehr komplexen Probleme bietet auch Ansätze für ganz alltägliche Aufgaben der diagnostischen Pathologie, z. B. der Nutzung der Daten aus Pathologiemanagementsystemen für ständig wachsende externe Anforderungen. Von Haroske $G$ et al., Dresden, wurde ein systematischer Ansatz für die Extraktion von strukturierten, maschinell handhabbaren Informationen aus pathohistologischen Befundtexten vorgestellt und mit einer Beispiellösung für kolorektale Tumoren unterlegt. Dieser Ansatz könnte einen Weg ebnen, die zahlreichen vorhandenen Dokumentationsanforderungen an Pathologen, wie sie durch die Tumordokumentation, aber auch durch Leitlinien u. a. der DGP und des Berufsverbandes vorgegeben sind, nahtlos in die praktisch flächendeckend vorhandenen Pathologiesysteme einzubinden und damit die Arbeit zu erleichtern. Inhaltlich gab es enge Beziehungen dieses Vortrages mit dem Hauptvortrag Fr-o1o (Brochhausen $M$ et al., Saarbrücken).

Die Sitzung der AG Informatik spiegelte einmal mehr recht anschaulich das wachsende Interesse der Pathologen am Verständnis für und der Anwendung von modernen Methoden der Informations- verarbeitung und Telematik in unserem Fachgebiet wider. Im Kontext der Sitzungen zur Systempathologie wird deutlich, wie stark die Entwicklung der Pathologie als Disziplin der medizinischen Wissenschaft von informationswissenschaftlichen Ansätzen und Methoden abhängig ist.

\section{Korrespondenzadresse \\ Prof. Dr. G. Haroske \\ Institut für Pathologie \\ Krankenhaus Dresden-Friedrichstadt \\ Friedrichstraße 41, 72076 Dresden \\ Gunter.Haroske@khdf.de}

Interessenkonflikt. Der korrespondierende Autor gibt an, dass kein Interessenkonflikt besteht. 